\title{
ASSESSMENT OF COGNITIVE FUNCTION, SOCIAL DISABILITY AND BASIC LIFE SKILLS IN EUTHYMIC PATIENTS WITH BIPOLAR DISORDER
}

\author{
Borjanka Batinic $^{1,2}$, Vanja Djokic ${ }^{1} \&$ Maja Ivkovic ${ }^{2,3}$ \\ ${ }^{1}$ Department of Psychology, Faculty of Philosophy, University of Belgrade, Belgrade, Serbia \\ ${ }^{2}$ Clinic of Psychiatry, University Clinical Centre of Serbia, Belgrade, Serbia \\ ${ }^{3}$ Medical School, University of Belgrade, Belgrade, Serbia
}

received: 15.10 .2020 ;

revised: 5.2.2021;

accepted: 2.3 .2021

\begin{abstract}
SUMMARY
Background: Data from the literature suggests the presence of cognitive impairments which persist in the euthymic phase of bipolar disorder $(B D)$ and have significant consequences in regards to psychosocial functioning. The aims of our study were: 1) to ascertain the cognitive function (CF), social disability (SD) and basic life skills (BLS) of euthymic patients diagnosed with BD, 2) their relationship and 3) to compare $C F, S D$ and BLS in euthymic patients diagnosed with BD to euthymic patients with recurrent major depressive disorder ( $r M D D)$.

Subjects and methods: Ninety eight euthymic patients diagnosed either with $B D(N=48$, mean age 48.79 years, $S D=8.587)$ or $r M D D(N=50$, mean age 50.02 years, $S D=9.826)$ underwent testing using the Brief Assessment of Cognition in Affective Disorders (BAC-A) test, the Sheehan Disability Scale (SDS) and the UCSD performance-based skills assessment (UPSA-brief).

Results: Euthymic patients with BD demonstrated significantly lower scores as compared to normal population values in verbal, learning and working memory, verbal fluency, attention and processing speed, affective memory for negative and positive words $(p \square 0.01$ each) and motor speed ( $p<0.05)$, but not for reasoning/problem solving $(p=0.05)$. Furthermore, their mean total SDS score of $17.60(S D=6.450, S k=-0.833)$ and its subscale scores were higher, while their UPSA-B total scores were lower $(M=76.01, S D$ $=17.148$, Sk $=-0.412)$. There was a correlation between $C F, S D$ and BLS scores $(p<0.01)$, as well as between BLS and $S D$ scores $(p<0.05)$. The analysis of variance did not however show significant differences between subgroups of patients.

Conclusion: Patients with euthymic BD had lower cognitive function, greater social disability and lower basic life skills. There were similar decreases in cognitive and psychosocial function between patients in the euthymic phase of either BD or MDD.
\end{abstract}

Key words: cognitive function - social disability - basic life skills - bipolar disorder

$* * * * *$

\section{INTRODUCTION}

Bipolar Disorder (BD) is a severe mental illness with significant psychosocial consequences for affected individuals and their families. Patients with BD experience noticeable cognitive and social impairment, not only during episodes, but also within the period of euthymia (Sarmento et al. 2020, Bortolato et al. 2015, Bourne et al. 2013, Torres et al. 2007). A systematic review of neurocognitive functioning in patients with BD revealed neurocognitive deficits in almost all cognitive domains (Esan et al. 2020, Tsitsipa \& Fountoulakis 2015). Patients with BD have dysfunctions in verbal and learning memory, working memory, motor speed, verbal fluency, executive functions, attention and speed of information processing and affective memory (Keefe et al. 2014, Mann-Wrobel et al. 2011, Arts et al. 2009, Bora et al. 2009). Significant general cognitive deficits are present during the first episode of BD and maintained during all subsequent stages, including the euthymic phase, with these deficits worsening during the course of the disorder (Toyshima et al. 2019, Szmulewicz et al. 2018, Mann-Wrobel et al. 2011, Levy et al. 2009, Bora et al. 2009, Robinson \& Ferrier 2006, Bearden et al. 2006). A meta-analysis by Buoli et al.
(2017) revealed that progression of changes in memory, executive domains and the prefrontal cortex during BD are similar to those seen in aging.

Cognitive impairment in BD has been shown to limit and impair work activity, social adjustment and quality of life during both the symptomatic and euthymic phases (Szmulewicz et al. 2018, Jensen et al. 2016, Baune \& Malhi 2015), as well as basic life skills and performance across all the different phases of the disorder (Henry et al. 2013). Neurocognitive impairments in verbal memory, executive functions and attention are the best long-term predictors of the psychofunctional outcome of BD during all phases of the disorder (Duarte et al. 2016, Bonnin et al. 2010, Martino et al. 2009). A meta-analysis by Depp et al. (2012) revealed a significant correlation between all cognitive domains and daily functioning in persons with $\mathrm{BD}$, with none of the examined moderators being significant (such as sample age, clinical state, and study design).

Comparisons of cognitive function in patients diagnosed with BD versus recurrent major depressive disorder (rMDD) have shown inconsistent results. Although both groups of patients show decreased cognitive performance compared to normal controls, when compared with each other older patients with BD show 
greater general deterioration in the areas of memory, executive functions, language skills and psychomotor skills (Gildengers et al. 2013). Bo et al. (2018) found that patients with $\mathrm{BD}$ had a significantly higher degree of cognitive impairment compared to patients with MDD. In a study by Maalouf et al. (2010) impairment in sustained attention was more specific to patients with bipolar affective disorder (both in the euthymic and depressive phases), while impairments in the domain of executive functions were more specific to unipolar depression and depressed states. On the contrary, a systematic review of comparative studies (Szmulewicz et al. 2017) could not provide data for distinctive neurocognitive profiles between patients with BD and MDD in the euthymic phase regarding attention and processing speed, executive functions and theory of mind, although there were some trends towards more impaired verbal memory in patients with $\mathrm{BD}$.

As such, the aims of our study were: 1) to ascertain the cognitive function, social disability and basic life skills in patients with euthymic BD, 2) to ascertain the mutual correlations between these factors and 3) to compare cognitive functioning, social disability and basic life skills in patients in the euthymic phase of either BD or rMDD. According to the aims of this study, we hypothesized the following:

- H1: In patients with euthymic BD, there would be a decrease in cognitive function compared to normal values in the following cognitive domains: verbal learning and memory, working memory, motor speed, verbal fluency, planning function (executive function), attention, information processing speed and affective memory.

- H2: Patients with euthymic BD would present with higher social disability and lower BLS compared to normal values.

- H3: There would be a correlation between cognitive efficiency, basic life skills and social disability in patients with euthymic BD, namely, patients with lower cognitive function would have a higher social disability and lower basic life skills.

- H4: Patients with euthymic BD would show decreased cognitive function, greater social disability and lower basic life skills as compared to patients with euthymic rMDD.

\section{SUBJECTS AND METHODS}

\section{Study sample}

A suitable study sample, consisting of 98 patients in the euthymic phase (48 patients diagnosed with BD and 50 patients diagnosed with rMDD), was recruited from the Clinic for Psychiatry, University Clinical Centre of Serbia. The diagnosis was made by attending psychiatrists, according to the International Classification of Diseases, Tenth Revision, and (ICD-10, World Health Organization 1992). Patients with comorbid substance abuse and CNS disorders were excluded. Both subgroups of patients were euthymic for at least one month. The study was approved by the Ethics Committee of the University Clinical Centre of Serbia, and written informed consent was obtained from all participants after a full description of the assessment measures.

\section{Assessment measures}

\section{The Brief Assessment of Cognition in Affective Disorders (BAC-A) (Keefe et al. 2014)}

The BAC-A test battery consists of 6 subtests for assessment of cognition in persons with affective disorders (verbal memory, working memory, motor speed, verbal fluency, planning function, attention and processing speed), and 1 additional test for affective processing (emotional distractibility and affective memory).

List learning (verbal memory): Fifteen words are presented to the patient, who is then asked to repeat as many of the words as they can remember. The procedure is repeated 5 times. The resulting score is the total number of memorized words for each time point (the total score ranges from $0-75$ points).

Digit sequencing tasks (working memory): Randomly ordered clusters of numbers of steadily increasing length are pronounced by the examiner, with the patients then asked to repeat the numbers from the smallest to the largest cluster. The resulting score is the total number of correct responses (the total score ranges from 0 to 28 points)

Token motor tasks (motor speed): Patients are given 100 plastic tokens and asked to put them as quickly as possible (two tokens at the same time) into the bowl over 60 seconds. The resulting score is the total number of tokens that are correctly placed in a bowl over 60 seconds (the total score ranges from 0-100 points).

Verbal fluency: Patients are asked to list as many words as possible within a given category (fruits) over 60 seconds. In the second part of this subtest, patients are also asked to list as many words as possible that begin with a particular letter over 60 seconds. This second subtest is repeated twice, with two different initial letters ( $\mathrm{F}$ and $\mathrm{S})$. The total number of words spoken over all three stages represents the overall score on this subtest.

Tower of London (executive function/reasoning and problem solving): Patients simultaneously view two images. Each image shows 3 differently coloured balls, placed differently on 3 pegs in each image. Patients are asked to determine the smallest number of moves of the balls in the first image that are required in order to arrange them identically to the balls in the second image. If the patients correctly determine the number of moves for 20 pairs of images, they are given two more pairs of pictures which are more difficult to solve than the previous 20 . The resulting score is the total number accurate responses (the total score ranges from 0-22 points). 
Symbol coding (attention and processing speed): At the beginning of the subtest, patients are given a response sheet in which a particular symbol matches to a particular number. Patients then write the numbers 1 to 9 as quickly as possible for 90 seconds so that they match the symbols in the answer sheet. The subtest is scored as the amount of numbers written correctly below the symbols (the total score ranges from 0-110 points).

Affective interference (affective distractibility and affective memory):

- In the first part of the subtest, 20 words are presented to patients, 10 of which are non-affective (fruits and vegetables), and 10 of which have high emotional valence, either positive (romantic) or negative (killer). Patients are given 3 trials to recall all the words they have heard. Then, they are asked to recall all the nonaffective words and all the affective words separately. The score for this first part of the subtest is $0-30$ points for non-selective words, and $0-15$ points for affective positive and affective negative words.

- The second part of the subtest is performed 15-20 minutes after the first part. Patients are read 10 nonaffective and 10 affective (5 positive and 5 negative) words they have already heard, and 10 non-affective and 10 affective ( 5 positive and 5 negative) words they have not previously heard. They then need to indicate whether they have already heard that word or not. This part of the subtest is measured as the following: the total number of accurate affective responses (0-10 points), the total number of accurate nonaffective responses (0-10 points), the total number of accurate affective (0-10 points) and accurate non-affective responses ( $0-10$ points) subtracted by the total number of inaccurate affective (0-10) and inaccurate non-affective responses (0-10). Although the patients performed both parts of this subtest during this study, the second part of the subtest was not used in the processing and interpretation of the results due to the lack of information regarding the interpretation of the second part of the test.

The BAC-A test proved to be useful for distinguishing between the patient and non-patient groups, for each of the 7 separate subtests: verbal memory, working memory, motor speed, verbal fluency, planning function, attention and processing speed, and affective memory. This test can be used for subjects aged 20 to 79 years. In our sample, this test demonstrated high internal consistency (Cronbach's $\alpha=0.848$ ). The average test time was between $30-45$ minutes, with the test being performed by a trained psychologist.

\section{The Sheehan disability scale (SDS) (Sheehan 1983)}

This scale is a self-assessment of social impairment in regards to work/school, social life, and family life/ responsibilities, and is measured by a 10 point visualanalogue scale (0-not at all, 1-3-mildly, 4-6-moderately, 7-9-markedly, 10-extremely). The total score is from 0 30 points, with higher scores associated with greater impairment. In our study, it showed high internal consistency $(\alpha=0.895)$.

\section{The UCSD Performance-Based Skill Assessment (UPSA-brief) (Patterson 2001)}

This is a role-playing test which assesses the basic life skills of a person in two selected areas: finance and communication. Patients who are tested receive props to demonstrate how they perform daily activities and are evaluated for their performance.

Subtest Finance, consists of two tasks: In the first task, the patient is provided by paper bills and metal coins and asked to return a correct amount of bills and coins based on a calculated amount. There are four tasks, with the first 3 assigned 1 point and the last assigned 2 points for correct answers. The second task involves filling out a blank check to pay a utility bill. The patient is given a real utility bill and is asked to fill out a check for the amount of money required to pay the bill. A total of six parts of the check need to be filled out with points given for each correct element. The overall score of this subtest ranges from 0-11 points. The reliability for this subtest was $\mathrm{r}=0.63$.

Subtest Communication: Patients are provided with an unplugged telephone and asked to role-play nine different scenarios. For example, in the first scenario the patient needs to correctly call the emergency number, 194, while the next scenario involves calling information to obtain a specific phone number, then dialling the number from memory. There are a total of nine communication subtasks. This subtest takes approximately 5 minutes. The overall score of this subtest ranges from 0-9 points. The reliability for this subtest was $\mathrm{r}=0.64$.

The raw scores of each subtest are added to get the total score, which ranges from $0-20$ points. The average time in which patients completed this test was 15 minutes. The reliability for the entire UPSA-brief test was $\mathrm{r}=0.73$.

\section{The semi-structured questionnaire of sociodemographic and clinical characteristics}

The semi-structured questionnaire covered issues relating to socio-demographic and clinical characteristics of the patients including age, education level and duration of illness.

\section{Statistical analysis}

The collected data were analysis using Statistical Package for the Social Sciences (IBM SPSS Statistics 22). The internal consistency of the study tests was determined by Cronbach's $\alpha$ test. For each questionnaire, a reliability analysis and a descriptive data analysis was performed. Descriptive analysis was also used to illustrate the socio-demographic and clinical characteristic of the sample. In order to test the hypotheses for comparison of cognition function in patients with BD versus normal control scores, new standardized scores were first made to include corrections for normal population data in the manual for the BAC-A tests. Subsequently, T-test was used to analyse all tests except for the SDS, which was presented as descriptive sta- 
tistics due to the nature of the test. Correlation analysis was used to determine correlations among cognitive, social functioning and basic life skills in persons with euthymic BD and rMDD.

\section{RESULTS}

\section{Descriptive statistics of the patient study sample}

The mean age of patients with BD was 48.79 years $(\mathrm{SD}=8.59)$, who were predominantly female $(70.8 \%)$, with maximum education levels of high school (54.2\%), college $(29.2 \%)$, or university $(16.7 \%)$. Mean duration of the disorder was 10.23 years $(\mathrm{SD}=5.64)$.

In the subsample of patients with rMDD, mean age was 50.02 years $(\mathrm{SD}=9.83)$, gender was predominantly female $(78.0 \%)$, with maximum education levels of high school $(54.0 \%)$, college $(28.0 \%)$, or university $(18.0 \%)$. Mean duration of the disorder was 9.04 years $(\mathrm{SD}=5.17)$.

\section{Cognitive function in patients with euthymic bipolar disorder assessed by BAC-A}

The $\mathrm{T}$ test for independent samples was used to compare the cognitive function of euthymic patients with BD to normal values. The analysis was carried out first by producing new, standardized patient scores for each subscale of the BAC-A test, using the following formula: Standard score $=($ score-M) $/$ SD. Mean $(\mathrm{M})$ and Standard Deviation (SD) of the normal population for each referring subscale was obtained from the BAC-A test manual. Then, the standardized scores for each subscale were then analyzed using a one-sample T test. These results are shown in Table 1. Patients with euthymic BD demonstra ted significantly lower scores as compared to normal values with the following cognitive measures: Verbal memory and learning $(\mathrm{M}=-1.50, \mathrm{SD}=1.02) ; \mathrm{t}(47)=-10.253$, $\mathrm{p}<0.01$; Motor speed $(\mathrm{M}=-0.52, \mathrm{SD}=1.20)$; $\mathrm{t}(47)=-2.977$, $\mathrm{p}<0.05$; Working memory $(\mathrm{M}=-1.39, \mathrm{SD}=1.15)$; $\mathrm{t}(47)=-$ $8.350, \mathrm{p}<0.01$; Verbal fluency $(\mathrm{M}=-1.10, \mathrm{SD}=1.20)$; $\mathrm{t}(47)=-6.517, \mathrm{p}<0.01$; Attention and speed of processing information $(\mathrm{M}=-1.71, \mathrm{SD}=1.15) ; \mathrm{t}(47)=-10.265, \mathrm{p}<0.01$; Affective memory, negative words $(\mathrm{M}=-0.62, \mathrm{SD}=1.15)$; $\mathrm{t}(47)=-3.733, \mathrm{p}<0.01$; Affective memory, positive words $(\mathrm{M}=-0.55, \mathrm{SD}=0.94) ; \mathrm{t}(47)=-4.122, \mathrm{p}<0.01$. The exception was executive function-reasoning/problem solving measured by the London Tower test $(\mathrm{M}=-0.12, \mathrm{SD}=1.50)$; $\mathrm{t}(47)=-0.558, \mathrm{p}=0.05$.

\section{Social disability in patients with euthymic bipolar disorder}

As shown in Table 2. Patients with euthymic BD had a mean score greater than 5.0 for each of the three aspects of the test. According to definitions by the authors of the scale (Sheehan 1983, Sheehan et al. 1996), our obtained means indicated the existence of disability in all three areas of social functioning: work/ school $(\mathrm{M}=5.21, \mathrm{SD}=2.43)$, social life/leisure activities $(\mathrm{M}=5.94, \mathrm{SD}=2.52)$, family life/home responsibilities $(\mathrm{M}=6.46, \mathrm{SD}=2.63)$, as well as in overall global social function $(\mathrm{M}=17.40, \mathrm{SD}=7.4)$. The distribution of individual and total score was negatively asymmetric, indicating that the respondents are more or less grouped at higher values, i.e. that they had significant impairment in terms of measures looking at single areas of function as well as in terms of overall global social function.

Table 1. Cognitive function in patients with euthymic bipolar disorder assessed by BAC-A

\begin{tabular}{|c|c|c|c|c|c|c|}
\hline Variables (BAC-A) & $\mathrm{N}$ & $\mathrm{M}$ & SD & $\mathrm{T}$ & $\mathrm{Df}$ & Significance \\
\hline Verbal memory /learning & 48 & -1.50 & 1.02 & -10.253 & 47 & $0.000 * *$ \\
\hline Motor speed & 48 & -0.52 & 1.20 & -2.977 & 47 & $0.005^{*}$ \\
\hline Working memory & 48 & -1.39 & 1.15 & -8.350 & 47 & $0.000 * *$ \\
\hline Verbal fluency & 48 & -1.10 & 1.20 & -6.517 & 47 & $0.000 * *$ \\
\hline Attention/speed of processing information & 48 & -1.71 & 1.15 & -10.265 & 47 & $0.000 * *$ \\
\hline Executivefunction & 48 & -0.12 & 1.50 & -0.558 & 47 & 0.580 \\
\hline Affective memory- negative words & 48 & -0.62 & 1.15 & -3.733 & 47 & $0.001 * *$ \\
\hline Affective memory- positive words & 48 & -0.55 & 0.94 & -4.122 & 47 & $0.000 * *$ \\
\hline
\end{tabular}

Table 2. Social disability in patients with euthymic bipolar disorder assessed by SDS

\begin{tabular}{lccccccc}
\hline Subscales (SDS) & N & Min & Max & M & SD & Sk & Ku \\
\hline Work/School & 48 & 0 & 10 & 5.21 & 2.43 & -0.624 & 0.674 \\
Social life/ Laisure activities & 48 & 0 & 10 & 5.94 & 2.52 & -0.791 & 0.433 \\
Family life /Home responsibilities & 48 & 0 & 10 & 6.46 & 2.63 & -0.947 & 0.193 \\
Total score & 48 & 0 & 30 & 17.40 & 7.40 & -0.943 & 0.562
\end{tabular}

Table 3. Basic life skills in patients with bipolar disorder in euthymic phase assessed by UPSA-brief

\begin{tabular}{lllllll}
\hline (UPSA-brief) & $\mathrm{N}$ & $\mathrm{M}$ & $\mathrm{SD}$ & $\mathrm{T}$ & Df & Significance \\
\hline Basic life skills - total score & 48 & -1.02 & 1.90 & -3.758 & 47 & $0.000^{*}$ \\
\hline${ }^{*} \mathrm{p}<0.01$ & & & & &
\end{tabular}




\section{Basic life skills in patients with bipolar disorder in euthymic phase}

The one-sample T test was used to compare basic life skills of patients with euthymic BD compared to normal values. The analysis was carried out by producing new, standardized patient scores from the overall score of the UPSA-brief using the following formula: Standard score $=($ score-M) $/ \mathrm{SD}$. The one-sample $\mathrm{T}$ test was chosen as the most appropriate analysis for comparing the two sample groups and testing the difference between them. These results are shown in Table 3 . Based on the results of the one-sample $\mathrm{T}$ test, there was a significant difference between euthymic persons with $\mathrm{BD}$ compared to the normal population in terms of basic life skills $(\mathrm{M}=-1.02, \mathrm{SD}=1.90) ; \mathrm{t}(47)=-3.758, \mathrm{p}<0.01$. Patients with euthymic BD have less capacity to deal with basic life skills as compared to the normal population.

\section{Correlation among cognitive function, social disability and basic life skills in patients diagnosed with euthymic bipolar disorder}

A correlation analysis was used to examine associations among cognitive function, social disability and basic life skills in patients diagnosed with euthymic BD for the three numerical test variables (scores on the SDS, the BAC-A and the UPSA-B). The results of this analysis are shown in Table 4. Correlation coefficient values indicate that there is a moderate correlation between basic life skills and social disability $(\mathrm{p}<0.05)$, and a high correlation between cognitive function and social disability $(\mathrm{p}<0.01)$ and cognitive functioning and basic life skills $(\mathrm{p}<0.01)$. Global social disability score did not correlate with the three measures of cognitive function (Motor speed, Positive Affective Memory and Negative Affective Memory), while the strongest correlation was with the scores on Verbal Memory and Learning $(\mathrm{p}<0.01)$.

\section{Comparison of cognitive function, social disability and basic life skills in patients with euthymic bipolar disorder versus euthymic recurrent depressive disorder}

As shown in Table 5, based on the Analysis of Variance, when the two group of patients were matched for age, gender, education and duration of disorder, there was no significant difference between the groups for the cognitive measures of motor speed and information processing speed $F(1)=1.192$, $p>0.05$, planning $F(1)=1.774$, $\mathrm{p}>0.05$, attention $\mathrm{F}(1)=0.000, \mathrm{p}>0.05$, working memory $\mathrm{F}(1)=1.090, \mathrm{p}>0.05$, verbal memory $\mathrm{F}(1)=2.248, \mathrm{p}>0.05$, and verbal fluency $F(1)=0.905, p>0.05$. Furthermore, there was no significant difference between the two groups for the total scores of basic life skills (UPSAbrief) $\mathrm{F}(1)=0.158, \mathrm{p}>0.05$, and social disability (SDS) $\mathrm{F}(1)=0.000, \mathrm{p}>0.05$.

Table 4. Correlation among cognitive function, social disability and basic life skills in patients diagnosed with euthymic bipolar disorder

\begin{tabular}{|c|c|c|c|c|c|c|}
\hline Variables & 1. & 2. & 3. & 4. & 5. & 6. \\
\hline Verbal learning and memory & 1 & 0.167 & $0.356^{*}$ & $0.510 * *$ & $0.558 * *$ & $0.366^{*}$ \\
\hline Motor speed & 0.167 & 1 & $0.442 * *$ & $0.526 * *$ & $0.503 * *$ & $0.581 * *$ \\
\hline Working memory & $0.356^{*}$ & $0.442 * *$ & 1 & $0.536 * *$ & $0.450 * *$ & $0.558 * *$ \\
\hline Attention /processing speed & $0.510 * *$ & $0.526 * *$ & $0.536 * *$ & 1 & $0.636 * *$ & $0.724 * *$ \\
\hline Verbal fluency & $0.558 * *$ & $0.503 * *$ & $0.450 * *$ & $0.636^{* *}$ & 1 & $0.687 * *$ \\
\hline Executive function & $0.366^{*}$ & $0.581 * *$ & $0.558 * *$ & $0.724 * *$ & $0.687 * *$ & 1 \\
\hline Afective memory -positive words & 0.200 & $0.433 * *$ & $0.403 * *$ & $0.571 * *$ & $0.486 * *$ & $0.539 * *$ \\
\hline Affective memory-negative words & 0.284 & $0.351 *$ & $0.443 * *$ & $0.5179 * *$ & $0.575 * *$ & $0.580 * *$ \\
\hline Basic life skills & $0.441 * *$ & $0.535 * *$ & $0.449 * *$ & $0.699 * *$ & $0.634 * *$ & $0.755^{* *}$ \\
\hline Social funkction & $-0.536 * *$ & -0.220 & $-0.340^{*}$ & $-0.358 *$ & $-0.368 *$ & $-0.376 * *$ \\
\hline \multirow[t]{2}{*}{ Cognitive function - total score } & $0.600 * *$ & $0.760 * *$ & $0.646 * *$ & $0.864 * *$ & $0.848 * *$ & $0.824 * *$ \\
\hline & & 7. & 8. & 9. & 10. & 11. \\
\hline Verbal learning and memory & & 0.200 & 0.284 & $0.441 * *$ & $-0.536 * *$ & $0.600 *$ \\
\hline Motor speed & & $0.433 * *$ & $0.351 *$ & $0.535 * *$ & -0.220 & $0.760 * *$ \\
\hline Working memory & & $0.403 * *$ & $0.443 * *$ & $0.449 * *$ & $-0.340^{*}$ & $0.646 * *$ \\
\hline Attention /processing speed & & $0.571 * *$ & $0.579 * *$ & $0.699 * *$ & $-0.358 *$ & $0.864 * *$ \\
\hline Verbal fluency & & $0.486 * *$ & $0.575 * *$ & $0.634 * *$ & $-0.368 *$ & $0.848 * *$ \\
\hline Executive function & & $0.539 * *$ & $0.580 * *$ & $0.755^{*} *$ & $-0.376^{* *}$ & $0.824 * *$ \\
\hline Afective memory -positive words & & 1 & $0.536 * *$ & $0.481 * *$ & -0.203 & $0.616^{* *}$ \\
\hline Affective memory-negative words & & $0.536 * *$ & 1 & $0.512 * *$ & -0.196 & $0.639 * *$ \\
\hline Basic life skills & & $0.481 * *$ & $0.512 * *$ & 1 & $-0.316^{*}$ & $0.762 * *$ \\
\hline Social funkction & & -0.203 & -0.196 & $-0.316^{*}$ & 1 & $-0.441 * *$ \\
\hline Cognitive function - total score & & $0.616 * *$ & $0.639 * *$ & $0.762 * *$ & $-0.441 * *$ & 1 \\
\hline
\end{tabular}
${ }^{*} \mathrm{p}<0.05 ;{ }^{* *} \mathrm{p}<0.01$ 
Table 5. Comparison of cognitive function, social disability and basic life skills in patients with euthymic bipolar disorder versus euthymic recurrent depressive disorder

\begin{tabular}{lcccc}
\hline & F & Df & Parcial eta squared & Significance \\
\hline Variables (BAC-A) & & & & 0.278 \\
$\quad$ Motor speed & 1.192 & 1 & 0.000 & 0.993 \\
Processing speed & 0.000 & 1 & 0.018 & 0.186 \\
Executive function & 1.774 & 1 & 0.000 & 0.993 \\
Atention & 0.000 & 1 & 0.011 & 0.299 \\
$\quad$ Working memory & 1.090 & 1 & 0.023 & 0.137 \\
$\quad$ Verbal learning and memory & 2.248 & 1 & 0.009 & 0.344 \\
Verbal fluency & 0.905 & 1 & & 0.512 \\
Variables (UPSA- brief) & & & 0.004 & 0.918 \\
Finance & 0.433 & 1 & 0.000 & 0.692 \\
Comunication & 0.011 & 1 & 0.002 & 0.309 \\
Total score & 0.158 & 1 & & 0.928 \\
Variables (SDS) & & & 0.011 & 0.289 \\
$\quad$ Work/school & 1.044 & 1 & 0.000 & 0.985 \\
$\quad$ Social life/leisure & 0.008 & 1 & 0.012 & 0.000 \\
$\quad$ Family life/responsibility & 1.136 & 1 & & \\
$\quad$ Total score & 0.000 & 1 & & \\
\hline
\end{tabular}

\section{DISCUSSION}

Our first hypothesis regarding the presence of cognitive deficits in euthymic patients diagnosed with BD as assessed by total BAC-A score, as well in terms of many different cognitive domains, was confirmed. Verbal learning and memory, verbal fluency as well as the speed of information processing were the most affected. These results are in line with data from previous studies (Tsitsipa \& Fountoulakis 2015, Bartolato et al. 2015, Torres et al. 2013). However, the patients from our sample did not show significant impairment in the executive function of reasoning and problem solving measured by the London Tower test, although there are numerous studies showing the opposite (Tsitsipa \& Fountoulakis 2015). A possible reason for this discrepancy was that there was no time limit for the London tower test, not allowing for a measure of reaction time which could lead to more detailed information about possible executive impairment. Furthermore, the corrections used in this study for comparison with the normal population are from the American population as provided by the author of the test. A Serbian population should be tested in future research, in order to make the results more trustworthy.

Our second hypothesis, that patients diagnosed with euthymic BD would be have higher social disability and lower basic life skills compared to the normal values, was confirmed as well. This is in line with previous meta-analyses of this topic (Szmulewicz et al. 2018, Jensen et al. 2016, Baune \& Malhi 2015, Henry et al. 2013).

The third hypothesis of this study, that there would be a correlation between cognitive function, social disability and basic life skills in patients with euthymic BD disorder, was also confirmed, as there was a statistically significant correlation among these depen- dent variables. However, the three measures of cognitive function-motor speed, affective memory of negative and of positive words, did not correlate with social disability. These results confirm that patients with euthymic BD and more cognitive impairments are also more dysfunctional in the social domain and less competent in terms of basic life skills. This is in line with previous studies which revealed significant disability and a strong correlation between disruptions of cognitive function and daily function that persisted during symptomatic periods as well as in euthymic periods (Duarte et al. 2018, Depp et al. 2012, Bonnin et al. 2010, Martino et al. 2009). When considering these results, a caveat is that some of the high and significant correlations that were obtained may be random as a consequence of the small study sample $(\mathrm{N}=48)$, as well as large number of dependent variables. In any event, the results of this study are consistent with the data from the literature indicating that persons with bipolar affective disorder, during periods of euthymia, experience significant impairment in cognition, social function and basic life skills. Although there was significant association among all three variables, the reason for less correlation of social disability with the other two variables may lie in the nature of the test itself, as it is a self-assessment questionnaire versus the independent assessment tests that measure cognitive impairment and basic life skills.

Our fourth hypothesis, that euthymic patients with BD would achieve worse cognitive function, higher social disability and lower basic life skills compared to euthymic patients with rMDD, was not confirmed, which is in concordance with results of the meta-analysis by Szmulewicz et al. (2017).

This research has contributed to a more general picture of the cognitive and social function and basic life skills of patients with euthymic BD. It is necessary 
to examine these constructs in a larger sample of patients with euthymic BD, and to compare the findings to the normal population in Serbia. Longitudinal studies could show the persistence and progression of changes in cognitive function, social function and basic life skills. Clinical variables including the classification of the euthymia and $\mathrm{BD}$ diagnoses (BD I, BD II), medication, premorbid IQ, history of psychosis and number of hospital admissions should also be included in the evaluation of the relationships among cognition, social disability and basic life skills.

\section{CONCLUSIONS}

The study results show that cognitive function, social function and basic life skills such as finance and communication are significantly impaired in euthymic patients with BDD compared to normal values, and also that greater cognitive impairment correlates with greater social disability and lower basic life skills. Euthymic patients with BD achieved results similar to those from euthymic patients with rMDD on the measures of cognitive function, social disability and basic life skills. The outcome for patients with $\mathrm{BD}$ and rMDD in psychosocial functioning could be improved by novel pharmacological and non-pharmacological individually customized treatments targeting neurocognitive deficits at the very beginning of these disorders (Sole et al. 2016, Filakovic et al. 2011).

\section{Acknowledgements:}

The authors would like to thank the patients who participated in the study, as well as Prof. Richard S.E. Keefe for granting the rights for use of the BAC-A.

\section{Conflict of interest: None to declare.}

\section{Contribution of individual authors:}

Borjanka Batinic: design of the study, literature research, writing introduction, interpretation of the data, writing conclusions;

Vanja Djokic: the sample collection and patient ratings, design of the study, statistical analysis, interpretation of the data;

Maja Ivkovic: the sample collection, test battery, interpretation of the data.

\section{References}

1. Arts B, Jabben N, Krabbendam L, van Os J: Meta-analyses of cognitive functioning in euthymic bipolar patients and their first-degree relatives. Correction. Psychol Med 2009; 39:525

2. Bearden CE, Glahn DC, Monkul ES, Barrett J, Najt P, Villarreal $V$, et al.: Patterns of memory impairment in bipolar disorder and unipolar major depression. Psychiatry Res 2006; 142:139-50
3. Baune BT, Malhi GS: A review on the impact of cognitive dysfunction on social, occupational, and general functional outcomes in bipolar disorder. Bipolar Disord 2015; 2:41-55

4. Bo Q, Dong F, Li X, Li F, Li P, Yu H, et al.: Comparison of cognitive performance in bipolar disorder, major depressive disorder, unaffected first-degree relatives, and healthy controls. Psychiatry Clin Neurosci 2019; 73:70-76

5. Bonnín CM, Martínez-Arán A, Torrent C, Pacchiarotti I, Rosa AR, Franco C, et al.: Clinical and neurocognitive predictors of functional outcome in bipolar euthymic patients: a long-term, follow-up study. J Affect Disord 2010; 121:156-60

6. Bora E, Yucel M, Pantelis C: Cognitive endophenotypes of bipolar disorder: a meta-analysis of neuropsychological deficits in euthymic patients and their first-degree relatives. J Affect Disord 2019; 113:1-20

7. Bora E, Yucel M, Pantelis C, Berk M: Meta-analytic review of neurocognition in bipolar II disorder. Acta Psychiatr Scand 2011; 123:165-174

8. Bourne C, Aydemir Ö, Balanzá-Martínez V, Bora E, Brissos S, Cavanagh JT, et al.: Neuropsychological testing of cognitive impairment in euthymic bipolar disorder: an individual patient data meta-analysis. Acta Psychiatr Scand 2013; 128:149-62

9. Bortolato B, Miskowiak KW, Köhler CA, Vieta E, Carvalho AF: Cognitive dysfunction in bipolar disorder and schizophrenia: a systematic review of meta-analyses. Neuropsychiatr Dis Treat 2015; 11:3111-25

10. Buoli M, Serati M, Caldiroli A, Cremaschi L, Altamura AC: Neurodevelopmental versus neurodegenerative model of schizophrenia and bipolar disorder comparison with psychological brain development and aging. Psychiatr Danub 2017; 29:24-27

11. Depp CA, Mausbach BT, Harmell AL, Savla GN, Bowie $C R$, Harvey PD, et al.: Meta-analysis of the association between cognitive abilities and everyday functioning in bipolar disorder. Bipolar Disord 2012; 14:217-26

12. Duarte W, Becerra R, Cruise K: The Relationship Between Neurocognitive Functioning and Occupational Functioning in Bipolar Disorder: A Literature Review. Eur J Psychol 2016; 12:659-678

13. Esan O, Oladele O, Adediran KI, Abiona TO: Neurocognitive Impairments (NCI) in bipolar disorder: Comparison with schizophrenia and healthy controls. J Affect Disord 2020; 277:175-181

14. Filakovic P, Petek Eric A, Pozgain P: New strategies in the treatment of bipolar disorder. Psychiatr Danub 2011; 23:293-299

15. Gildengers AG, Chisholm D, Butters MA, Anderson SJ, Begley A, Holm M, et al.: Two-year course of cognitive function and instrumental activities of daily living in older adults with bipolar disorder: evidence for neuroprogression? Psychol Med 2013; 43:801-11

16. Henry BL, Minassian A, Perry W: Everyday functional ability across different phases of bipolar disorder. Psychiatry Res 2013; 210:850-6

17. Keefe RS, Fox KH, Davis VG, Kennel C, Walker TM, Burdick KE, et al.: The brief assessment of cognition in affective disorders (BAC-A): performance of patients with bipolar depression and healthy controls. J Affect Disord 2014; 166:86-92

18. Jensen JH, Knorr U, Vinberg M, Kessing LV, Miskowiak $K W$ : Discrete neurocognitive subgroups in fully or 
partially remitted bipolar disorder: Associations with functional abilities. J Affect Disord 2016; 205:378-386

19. Maalouf FT, Klein C, Clark L, Sahakian BJ, La Barbara, EJ, Versace A, et al.: Impaired sustained attention and executive dysfunction: bipolar disorder versus depressionspecific markers of affective disorders. Neuropsychologia 2010; 48:1862-1868

20. Mann-Wrobel MC, Carreno JT, Dickinson D: Meta-analysis of neuropsychological functioning in euthymic bipolar disorder: an update and investigation of moderator variables. Bipolar Disord 2011; 13:334-342

21. Martino DJ, Marengo E, Igoa A, Scápola M, Ais ED, Perinot $L$, et al.: Neurocognitive and symptomatic predictors of functional outcome in bipolar disorders: a prospective 1 year follow-up study. J Affect Disord 2009; 116:37-42

22. Patterson TL: Meta-analysis of the association between cognitive abilities and everyday functioning in bipolar disorder. Bipolar Disord 2012; 14:217-226

23. Robinson LJ, Ferrier IN: Evolution of cognitive impairment in bipolar disorder: a systematic review of crosssectional evidence. Bipolar Disord 2006; 8:103-16

24. Sarmento SMS, Bittencourt L, de Mendonça Filho EJ, Abreu N, Tavares de Lacerda AL, Miranda-Scippa Â: Neurocognitive Impairment in Bipolar Disorder and Associated Factors: Using Population-based Norms and a
Strict Criterion for Impairment Definition. Cogn Behav Neurol 2020; 33:103-112

25. Szmulewicz AG, Valerio MP, Smith JM, Samamé $C$, Martino DJ, Strejilevich SA: Neuropsychological profiles of major depressive disorder and bipolar disorder during euthymia. A systematic literature review of comparative studies. Psychiatry Res 2017; 248:127-133

26. Szmulewicz AG, Valerio MP, Lomastro J, Smith JM, Chiappe V, Martino DJ, et al.: Neurocognitive functioning in first-episode Bipolar Disorder: Relationship with functional status. J Affect Disor 2018; 228:97-100

27. Torres IJ, Boudreau VG, Yatham LN: Neuropsychological functioning in euthymic bipolar disorder: a meta-analysis. Acta Psychiatr Scand Suppl 2007; 434:17-26

28. Toyoshima K, Kako Y, Toyomaki A, Shimizu Y, Tanaka T, Nakagawa S, Inoue T, Martinez-Aran A, Vieta E, Kusumi I. Associations between cognitive impairment and quality of life in euthymic bipolar patients. Psychiatry Res 2019; 271:510-515

29. Tsitsipa E: The neurocognitive functioning in bipolar disorder: a systematic review of data. Ann Gen Psychiatry $2015 ; 14: 42$

30. Tsitsipa E, Fountoulakis KN: The neurocognitive functioning in bipolar disorder: a systematic review of data. Ann Gen Psychiatry; 1; 14:42

Correspondence:

Associate Professor Borjanka Batinic, MD, PhD

Department of Psychology, Faculty of Philosophy, University of Belgrade

Cika Ljubina 18-20, 11 000, Belgrade, Serbia

E-mail:doubleb@eunet.rs 